\title{
First report of cerebellar abiotrophy in an Arabian foal from Argentina
}

\author{
S.A. Sadaba ${ }^{1,2}$, G.J. Madariaga ${ }^{3}$, C.M. Corbi Botto ${ }^{1,2}$, M.H. Carino' ${ }^{1}$ M.E. Zappa ${ }^{1}$, P. Peral García ${ }^{1}$, S.A. Olguín ${ }^{4}$, \\ A. Massone ${ }^{3}$ and S. Díaz ${ }^{1, *}$ \\ ${ }^{1}$ IGEVET - Instituto de Genética Veterinaria "Ing. Fernando Noel Dulout" (UNLP-CONICET La Plata), Facultad \\ de Ciencias Veterinarias, Universidad Nacional de La Plata, La Plata, Argentina \\ ${ }^{2}$ Research Fellows from Consejo Nacional de Investigaciones Científicas y Técnicas (CONICET). Av. Rivadavia \\ 1917 (C1033AAJ) CABA, Argentina \\ ${ }^{3}$ Laboratorio de Patología Especial Veterinaria, Facultad de Ciencias Veterinarias, Universidad Nacional de La \\ Plata, La Plata, Argentina \\ ${ }^{4}$ Cátedra de Métodos Complementarios de Diagnóstico, Facultad de Ciencias Veterinarias, Universidad Nacional \\ de La Plata, La Plata, Argentina
}

\begin{abstract}
Evidence of cerebellar abiotrophy (CA) was found in a six-month-old Arabian filly with signs of incoordination, head tremor, wobbling, loss of balance and falling over, consistent with a cerebellar lesion. Normal hematology profile blood test and cerebrospinal fluid analysis excluded infectious encephalitis, and serological testing for Sarcocystis neurona was negative. The filly was euthanized. Postmortem X-ray radiography of the cervical cephalic region identified not abnormalities, discounting spinal trauma. The histopathological analysis of serial transverse cerebellar sections by electron microscopy revealed morphological characteristics of apoptotic cells with pyknotic nuclei and degenerate mitochondria, cytoplasmic condensation and areas with absence of Purkinje cells, matching with CA histopathological characteristics. The indirect DNA test for CA was positive in the filly, and DNA test confirmed the CA carrier state in the parents and the recessive inheritance of the disease. To our knowledge this is the first report of a CA case in Argentina.
\end{abstract}

Keywords: Apoptosis, Arabian horses, Cerebellar abiotrophy, DNA, Purkinje cells.

\section{Introduction}

In the Arabian horse, head injuries along with cerebellar abiotrophy (CA) and meningoencephalitis are the most common processes affecting the cerebellum. For differential CA diagnosis, family congenital diseases such as cerebellar hypoplasia, diseases caused by physical head injuries, meningoencephalomyelitis of diverse etiology, toxic diseases (mycotoxins) and neoplasms should be discarded by a series of analysis.

$\mathrm{CA}$ is a neurological condition which affects a number of animal species characterized by postnatal degeneration of Purkinje cells (de Lahunta, 1990). Although the symptoms and pathological features of this neurodegenerative condition are the same as in other species, in horses, CA is mainly found in the Arabian horse breed (Fraser, 1966; Blanco et al., 2006), and it has also been described in Swedish Gotland ponies and in an American Miniature Horse colt (Fox et al., 2000).

The occurrence of CA in other horse breeds is not wellknown, because breeders are usually reluctant to disclose that their breeding stock has produced foals with a neurological disease (Brault and Penedo, 2011).
New DNA scanning techniques have identified at least one CA carrier in three additional breeds, such as Bashkir Curly Horses, Trakehners and Welsh ponies, with a CA allele frequency between 2.8 and $0.33 \%$ (Brault and Penedo, 2011). As pointed out by these authors, CA was introduced into these breeds by Arabian ancestry. Therefore, the CA mutation is present in horse breeds that allow crossbreeding with Arabian horses and in breeds that have used Arabians as foundation stock during their development (Brault $e t$ al., 2011b). To our knowledge this is the first report of a CA case in Argentina. Therefore, the risk of producing an affected foal from a particular mating is still unknown.

CA is characterized by postnatal (between six weeks and four months of age) degeneration of cerebellar Purkinje cells. Since Purkinje cells are the most prominently and consistently affected component, their degeneration leads to a concurrent degeneration of cerebellar granule neurons (Dungworth and Fowler, 1966; Fraser, 1966; Palmer et al., 1973) and to a disorganization of the molecular and granular layers, with the remaining Purkinje cells being small and shrunken. 
Overall size and thickness of the cerebellum, however, is largely unaffected (Dungworth and Fowler, 1966). Without Purkinje cells, the horse loses spatial and time perception, making it difficult to maintain the equilibrium and the coordination of the body members. Although CA itself is not fatal, the general lack of balance and hyperactivity of affected horses poses a danger to both horses and handlers (Brault and Penedo, 2011). Symptoms include intention head tremors, ataxia, exaggerated or paddling action of the forelegs, a wide-based stance and a lack of menace response (Dungworth and Fowler, 1966; Palmer et al., 1973; DeBowes et al., 1987).

CA-affected horses are often confused with those affected with wobbler, which is a condition of the spinal cord, not of the brain, or are misdiagnosed as animals that have suffered a head injury in an accident. Although symptoms are distinctive, they are similar to other neurological conditions; thus, accurate diagnosis can be a challenge and is often reached only after other possible conditions have been eliminated (Dungworth and Fowler, 1966; Brault et al., 2011b). Until recently, conclusive diagnosis of the disease could only be made postmortem by histopathological exam of the cerebellum after euthanasia.

CA is inherited as an autosomal recessive trait (Brault et al., 2011b). Preliminary data suggest an approximate location for $\mathrm{CA}$ on the horse genome, indicating the presence of a gene in this region that may be responsible for $\mathrm{CA}$. A single nucleotide polymorphism (CA allele) has been identified in the horse TOE1 gene which is associated with CA in Arabian horses (Brault et al., 2011a).

A test has now been developed which can be used to identify affected foals; in addition, it would be possible to determine if a horse carries one or two copies of the CA allele, using different tissues. Test results are reported as "N", meaning that the mutation associated with CA is not present, or "CA", indicating the presence of the CA mutation. The test is available in most horse genetics molecular diagnostic laboratories.

This case report describes the presence of ultrastructural signs of apoptosis in Purkinje cells of an Arabian filly affected with CA, and the complementary diagnostic methods employed to accurately determine the CA status for the first time in a foal in Argentina.

\section{Case details}

A 6-month-old Arabian filly was presented to the responsible veterinarian with a history of progressive clinical signs of loss of balance, head tremor and wobbling. There was no history of trauma or previous illness. The animal was from a closed breeding herd of Arabian horses with no history of similar cases, exhibiting progressive neurologic abnormalities consistent with cerebellar disease first noticed at weaning. Physical examination revealed the colt to be alert and responsive but exhibiting head tremor and a base-wide stance in the forelimbs. Pulse rate, respiratory rate and temperature were within normal limits. The colt had a tendency to fall to the side or back, and spastic hyperextension of the forelimbs when it was startled. There was no evidence of neuromuscular weakness. Clinical signs were consistent with a cerebellar lesion.

Hematology profile blood test values were normal, and cerebrospinal fluid analysis (CSF) was unremarkable, thus allowing exclusion of infectious encephalitis. Serological reaction tests for Sarcocystis neurona were negative. On the other hand, X-rays of the cervical region showed no abnormalities, discounting spinal trauma. The CA-affected filly was euthanized. Postmortem X-ray scan of the cervical cephalic region identified no abnormalities. Dorsoventral (DV) radiographic views showed an unaltered atlantooccipital space. Both the lateral left-side (LLI) and the DV views showed normal cervical disc spaces, like the vertebral canal, with good spinal alignment and no signs of listhesis.

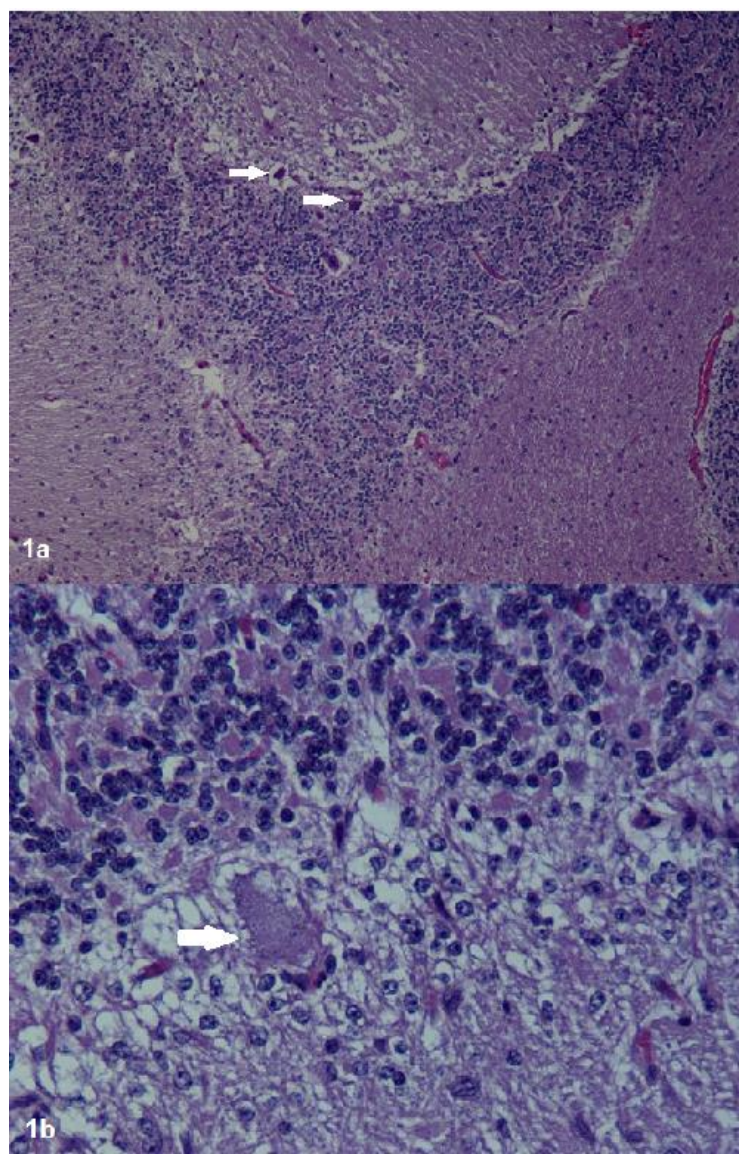

Fig. 1. Histological examination of cerebellar sections after staining with hematoxylin and eosin. a: Cerebellum of the CA affected foal $10 \mathrm{x}$ : Almost complete absence of Purkinje cells. The remaining Purkinje cells are shrunken and hyperchromatic. b: Cerebellum of the CA affected foal $40 \mathrm{x}$. 
The cerebellum was removed and fixed. Histopathological studies (Fig 1a,b) of serial transverse cerebellar sections and electron microscopy (JEM $1200 \mathrm{EX}$ II, Jeol), revealed morphological characteristics of apoptotic cells with pyknotic nuclei and degenerate mitochondria, cytoplasmic condensation and areas with absence of Purkinje cells, allowing us to confirm the CA diagnosis (Fig 2a,b,c). The indirect DNA test for CA was positive in the filly, and DNA testing of the parents confirmed their carrier state and the recessive inheritance of the disease.

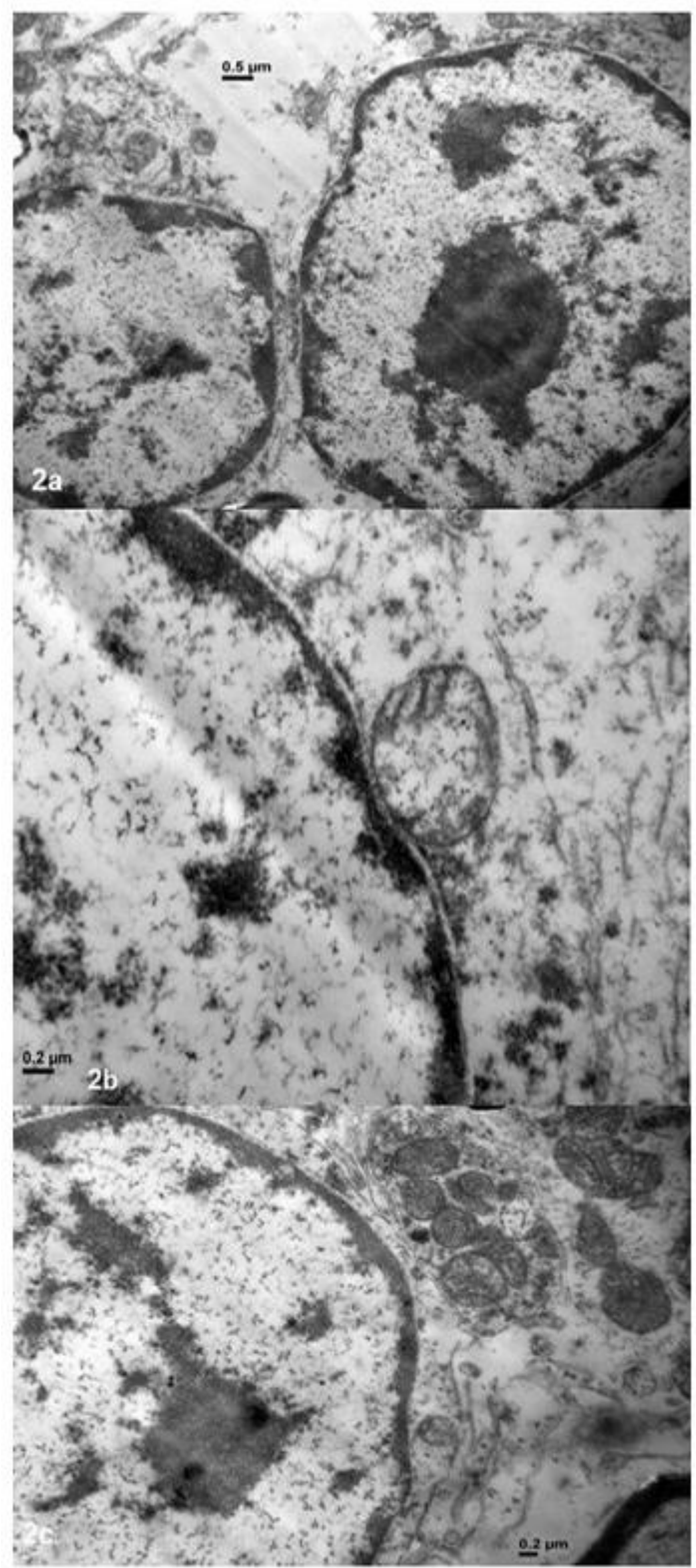

Fig. 2. Electron microscopy (JEM 1200EX II, Jeol) of a cerebellar section from the CA-affected foal (Camera ES500W Erlangshen C). (a): Morphological characteristics of apoptotic cells $(0.5 \mu \mathrm{m})$. (b-c): degenerate mitochondria, pale, swollen and vacuolated Purkinje cells $(0.2 \mu \mathrm{m})$.

\section{Discussion}

The complementary methods employed in this study to make an accurate diagnosis of CA were effective. Since $\mathrm{CA}$ is inherited as a recessive trait, the mating of two carriers may result in an affected foal (Brault et al., 2011b). Thus, for a foal to be born with CA, two copies of the mutated allele must be present, each corresponding to each parent. Only mating between two carriers can produce an affected foal. Carriers of the mutant allele (heterozygous) do not have clinical signs, and the carrier state does not have negative consequences for health and athletic performance. The indirect DNA test for CA was positive in the filly, and DNA testing of both parents confirmed their carrier state, consequently the mating of the two heterozygote parents resulted in an affected foal.

CA diagnosis in the Arab population would be useful for Arabian breeders from our country when choosing their breeding stock (Brault et al., 2011b; Brault and Penedo, 2011). To date it is only relevant whether the animals concerned are known to have produced affected foals, in order to eliminate a genetic disorder. To our knowledge this is the first case reported of a CA case in Argentina. The Arabian filly with CA belongs to a closed breeding herd of Arabian horses with no previous history of similar clinical signs of cerebellar defects. A second foal was CA diagnosed in the same year, but the observed clinical signs were slightly present, and could be confused with other conditions. This second foal descends from the same stallion and a different carrier dam. These two foals were the first cases of the disease in the history of the farm.

Since no CA cases were reported to date in Argentina, the risk of producing an affected foal from a particular mating is still unknown. The complementary diagnosis methods employed here allowed to accurate CA diagnosis, which was confirmed by the DNA test. The molecular test also allowed determining the carrier state of the stallion and the dam, giving the breeder the knowledge for choosing the matings in order to diminish the probabilities of produce affected foals.

\section{Conflict of interest}

The authors declare that there is no conflict of interest.

\section{Acknowledgements}

The authors wish to acknowledge the Electronic Microscopy Service from the National University of La Plata School of Veterinary Sciences for their assistance in acquiring the 6-month-old foal cerebellum sample. We are also grateful to the Arabian owner and breeder who generously provided the filly and DNA samples of the parents for research purposes. Thanks are also due to A. Di Maggio for manuscript correction.

\section{References}

Blanco, A., Moyano, R., Vivo, J., Flores-Acuña, R., Molina, A., Blanco, C. and Monterde JG. 2006. 
Purkinje cell apoptosis in Arabian horses with cerebellar abiotrophy. J. Vet. Med. A Physiol. Pathol. Clin. Med. 53, 286-287.

Brault, L.S., Cooper, C.A., Famula, T.R., Murray, J.D. and Penedo, M.C. 2011a. Mapping of equine cerebellar abiotrophy to ECA2 and identification of a potential causative mutation affecting expression of MUTYH. Genomics 97, 121-129.

Brault, L.S., Famula, T.R. and Penedo, M.C. 2011 b. Inheritance of equine cerebellar abiotrophy in Arabians. Am. J. Vet. Res. 72(7), 940-944.

Brault, L.S. and Penedo, M.C. 2011. The frequency of the equine cerebellar abiotrophy mutation in nonArabian horse breeds. Equine Vet. J. 43(6), 727731.

DeBowes, R.M., Leipold, H.W. and Turner-Beatty, M. 1987. Cerebellar abiotrophy. Vet. Clin. North Am.
Equine Pract. 3(2), 345-352.

de Lahunta, A. 1990. Abiotrophy in domestic animals: a review. Can. J. Vet. Res. 54(1), 65-76.

Dungworth, D.L. and Fowler, M.E. 1966. Cerebellar hypoplasia and degeneration in a foal. Cornell Vet. 56(1), 17-24.

Fox, J., Duncan, R., Friday, P., Klein, B. and Scarratt, W. 2000. Cerebello-olivary and lateral (accessory) cuneate degeneration in a juvenile American Miniature Horse. Vet. Pathol. 37(3), 271-274.

Fraser, H. 1966. Two dissimilar types of cerebellar disorder in the horse. Vet. Rec. 78(18), 608-612.

Palmer, A.C., Blakemore, W.F., Cook, W.R., Platt, H. and Whitwell, K.E. 1973. Cerebellar hypoplasia and degeneration in the young Arab horse: clinical and neuropathological features. Vet. Rec. 93(3), 6266. 